\title{
REGULARITY FOR DEGENERATE EVOLUTION EQUATIONS WITH STRONG ABSORPTION
}

\author{
JOÃO VITOR DA SILVA, PABLO OCHOA \\ \& \\ ANALIA SILVA
}

\begin{abstract}
In this manuscript, we study geometric regularity estimates for degenerate parabolic equations of p-Laplacian type $(2 \leq p<\infty)$ under a strong absorption condition:

$$
\Delta_{p} u-\frac{\partial u}{\partial t}=\lambda_{0} u_{+}^{q} \quad \text { in } \quad \Omega_{T}:=\Omega \times(0, T),
$$

where $0 \leq q<1$. This model is interesting because it yields the formation of dead-core sets, i.e, regions where nonnegative solutions vanish identically. We shall prove sharp and improved parabolic $C^{\alpha}$ regularity estimates along the set $\mathfrak{F}_{0}\left(u, \Omega_{T}\right)=\partial\{u>0\} \cap \Omega_{T}$ (the free boundary), where $\alpha=\frac{p}{p-1-q} \geq 1+\frac{1}{p-1}$. Some weak geometric and measure theoretical properties as non-degeneracy, positive density, porosity and finite speed of propagation are proved. As an application, we prove a Liouville-type result for entire solutions. A specific analysis for Blow-up type solutions will be done as well. The results are new even for dead-core problems driven by the heat operator.
\end{abstract}

\section{INTRODUCTION}

Throughout the last 40 years a wide class of parabolic equations has been used to model problems coming from chemical reactions, physical-mathematical phenomena, biological processes, population dynamics, among others. Some of the main topics approached are reaction-diffusion processes with one-phase transition. Thus, the existence of non-negative solutions plays an essential role in these studies (cf. [7], [28] and references therein). An enlightening prototype is the following model of an isothermal catalytic reaction-diffusion process:

$$
\left\{\begin{aligned}
\Delta u-\frac{\partial u}{\partial t} & =f(u) \quad \text { in } \quad \Omega_{T} \\
u(x, t) & =g(x, t) \quad \text { on } \partial \Omega \times(0, T) \\
u(x, 0) & =u_{0}(x) \quad \text { in } \bar{\Omega},
\end{aligned}\right.
$$

where the boundary data fulfils

$$
0<u_{0} \in C^{0}(\bar{\Omega}), g(x, t)=\mathfrak{j}>0 \quad \text { and } \quad u(x, 0)=\mathfrak{j} \forall x \in \partial \Omega .
$$

Here, $u$ represents the concentration of a reactant evolving in time, where $\Omega_{T}:=\Omega \times(0, T), \Omega \subset \mathbb{R}^{N}$ is a regular and bounded domain and $f$ is a non-linear convection term fulfilling $f(s)>0$ if $s>0$ and $f(0)=0$. Moreover, the boundary conditions mean that the reactant is injected with a constant isothermal flux on the boundary. Recall that in evolution problems when $f$ is locally Lipschitz, it follows from the Maximum Principle that non-negative solutions must be strictly positive. However when $f$ is not Lipschitz (or not decaying sufficiently fast) at the origin, then non-negative solutions may exhibit a plateau region, which is known in the literature as Dead-Core set, i.e., a region of positive measure where non-negative solutions vanish identically.

Advances in connection with existence theory of dead-core solutions, formation of dead-core regions and decay estimates at infinity were obtained in [7], [21] and [27]. Other properties of solutions such as growth of interfaces, shrink and estimates of/on the support and finite extinction in reaction-diffusion problems together with other qualitative properties may be found in Díaz et al's fundamental articles [1], [3], [16], [17], the Antontsev et al's classical book [4], the survey [15] and references therein. However, the lack of quantitative properties for

2000 Mathematics Subject Classification. 35B53, 35B65, 35J60, 35K55, 35K65.

Key words and phrases. $p$-Laplacian type operators, dead-core problems, sharp and improved intrinsic regularity, Liouville type results. 
$p$-parabolic dead-core problems constitutes our starting point in this research. In particular, we shall be interested in the derivation of quantitative results for the following class of parabolic dead-core problems of p-Laplacian type:

$$
\Delta_{p} u-\frac{\partial u}{\partial t}=\lambda_{0}(x, t) \cdot u_{+}^{q}(x, t) \quad \text { in } \quad \Omega_{T},
$$

with suitable boundary data, where $u_{+}=\max \{u, 0\}, 0 \leq q<1, p \geq 2, \Omega \subset \mathbb{R}^{N}$ is a bounded smooth domain and $\lambda_{0}$ (the Thiele modulus) is bounded away from zero and infinity. We refer the reader to Section 2 for notation and definitions.

An important feature in free boundary problems consists of Non-degeneracy of solutions (cf. [11], [12], [13], [25] and [31] for some examples in elliptic and parabolic settings). Our first main result gives the precise rate at which weak solutions $u$ to (1.2) detach from their free boundaries.

Theorem 1.1 (Non-degeneracy). Let $u$ be a weak solution to (1.2). Then for every $\left(x_{0}, t_{0}\right) \in \overline{\{u>0\}}$ and $r>0$ such that $Q_{r}\left(x_{0}, t_{0}\right) \Subset \Omega_{T}$ there holds:

$$
\sup _{\partial_{p} Q_{r}^{-}\left(x_{0}, t_{0}\right)} u(x, t) \geq \mathfrak{C}_{0}^{*} \cdot r^{\frac{p}{p-q-1}}
$$

for a universal constant $\mathfrak{C}_{0}^{*}>0$.

Throughout this paper universal constants are the ones depending only on dimension and structural properties of the problem, i. e. $N, p, q$ and bounds of $\lambda_{0}$.

The proof of Theorem 1.1 consists of combining the construction of an appropriate barrier function

$$
\Phi(x, t)=\mathfrak{a} \cdot\left(|x|^{\frac{p}{p-1}}+\mathfrak{b} t^{\frac{p-1-q}{(p-1)(1-q)}}\right)^{\frac{p-1}{p-1-q}},
$$

for universal constants $\mathfrak{a}, \mathfrak{b}>0$, together with a version of comparison principle to dead-core solutions. Observe that the assumption $0 \leq q<1$ is necessary to guarantee that the barrier does not blow up as $t$ tends to 0 .

We also establish an upper growth estimate for dead-core solutions of (1.2).

Theorem 1.2 (Improved regularity along free boundary). Let $u$ be a non-negative and bounded weak solution to (1.2), such that $\frac{\partial u}{\partial t} \geq 0$ a.e. in $\Omega_{T}$. Then, for every compact set $\mathrm{K} \Subset \Omega_{T}$ and for every $\left(x_{0}, t_{0}\right) \in \partial\{u>0\} \cap \mathrm{K}$, there holds:

$$
u(x, t) \leq \mathfrak{C} .\|u\|_{L^{\infty}\left(\Omega_{T}\right)}\left(\left|x-x_{0}\right|+\left|t-t_{0}\right|^{\frac{1}{\theta}}\right)^{\frac{p}{p-q-1}},
$$

for a constant $\mathfrak{C}>0$ depending only on universal parameters and $\operatorname{dist}\left(\mathrm{K}, \partial_{p} \Omega_{T}\right)$ and for all $(x, t)$ sufficiently close to $\left(x_{0}, t_{0}\right)$.

We briefly discuss some heuristic remarks related to the content of Theorem 1.2. Firstly, the assumption of monotonicity in time is natural in the literature, see for example [12], [25] and [26], and it is not restrictive, because it can be deduced from the boundary conditions, for example.

Recall that the lack of Strong Minimum Principle, namely when $q<1$, might lead to the existence of plateau regions to solutions of (1.2). In effect, the following profiles

$$
u(x, t):=\left[(1-q) \lambda_{0} \cdot\left(t_{0}-t\right)\right]_{+}^{\frac{1}{1-q}} \quad \text { or } \quad u(x, t):=\left[\lambda_{0} \cdot \frac{(p-q-1)^{p}}{p^{p-1}(p q+N(p-1-q))}\right]^{\frac{1}{p-q-1}} \cdot( \pm x)_{+}^{\frac{p}{p-q-1}}
$$

are weak dead-core solutions to (1.2) with $\lambda_{0}(x, t)=\lambda_{0}$. This shows that that dead-core solutions may exist. With respect to regularity, let us recall that in general (bounded) solutions to (1.2) are parabolically $C^{1+\alpha}$ for some $0<\alpha \leq 1$ (cf. [18], [20], and [34]). Furthermore, a local $C^{\alpha^{\sharp}}$ behaviour for $\alpha^{\sharp}=1+\frac{1}{p-1}$ is the better regularity estimates expected for weak solutions with bounded right hand side (compare with [2] in the context of elliptic and parabolic $p$-obstacle problems and $[5,6]$ for the elliptic context of the so termed $C^{p^{\prime}}$ regularity conjecture). 
Notice that the dead-core analysis carries on an insightful and deep significance: solutions to (1.2) have an improved pointwise behaviour as following:

$$
\sup _{Q_{r}\left(x_{0}, t_{0}\right)} u(x, t) \approx r^{\alpha_{0}(p, q)} \text { for } \quad \alpha_{0}(p, q):=\frac{p}{p-q-1},
$$

around free boundary points. Therefore, Theorem 1.2 yields that solutions are expected to be $C_{x, t}^{\left\lfloor\alpha_{0}\right\rfloor, \frac{\left\lfloor\alpha_{0}\right\rfloor}{\theta}}$ at free boundary points, where $\theta$ is the intrinsic scaling factor in time variable:

$$
\theta=\theta\left(p, \alpha_{0}\right):=p+\alpha_{0}(2-p) .
$$

We point out that for $p \geq 2$ :

$$
\theta(p, q)=\frac{p(1-q)}{p-q-1}>0 \quad \Leftrightarrow \quad 0 \leq q<1,
$$

which assures once again that the assumption $0 \leq q<1$ must be present. Moreover, notice that:

$$
\alpha_{0}=\frac{p}{p-q-1}>1+\frac{1}{p-1}=\alpha^{\sharp} \Leftrightarrow q>0 .
$$

Thus, we derive better regularity estimates along $\partial\{u>0\} \cap \Omega_{T}$. Also, observe that $\alpha_{0}>2$ provided $2 \leq p<4$, i.e., weak solutions become classical solutions. In summary, thanks to Theorem 1.2 we can access better regularity estimates on free boundary points than those available currently (cf. [5, 6], [18], [20] and [34]). It is worth highlighting that such an approach has become a mainstream line of investigation in the literature. We must mention, as one of the starting points of this theory, the Teixeira's work [29] where improved regularity estimates are obtained in the context of degenerate elliptic PDEs precisely along their set of critical points.

The technique applied in the proof of Theorem 1.2 can be adjusted in order to yield other relevant consequences. In this direction, we are able to establish a sharp gradient decay at free boundary points for a particular class of dead-core solutions, see Lemma 3.8 for more details.

Our article has been strongly influenced by the geometric tangential analysis, intrinsic scaling technique and the theory of geometric free boundary problems (a list of surveys related to these topics is [8], [9], [10], [14], [23], [24], [26], [30], [32], [33], and [34]). Particularly, we should quote [22], [25] and [31], which are groundbreaking manuscripts in the modern theory of free boundary and dead-core problems. Some of the core ideas further developed in this current article were introduced in such pivotal references.

We point out some final comments. The main obstacle in obtaining the estimates from Theorem 1.2 for the singular case $1<p<2$ is that the technique applied to prove the Non-degeneracy property, does not work for the singular setting. Finally, we highlight that the methods employed through this paper only explore the structure and intrinsic scaling of the $p$-Laplacian operator. Indeed, the only requirement is the compactness argument, a priori estimates and the strong maximum principle for the limiting equation. Consequently, the proofs can be adapted to more general parabolic problems of $p$-Laplacian type.

Our article is organized as following: In Section 2 we shall present some preliminaries tools. An appropriated notion of weak solution is enunciated. We will also refer some results about comparison and we introduce a family of solutions invariant under intrinsic scaling. In Section 3, we will prove a central result in our article, Lemma 3.1, which allows us to place solutions in a flatness improvement regime. At the end of this section, we prove Theorem 1.2. Similar growth rate for gradient along free boundary points will also be performed, Lemma 3.8. Section 4 will be devoted to prove Non-degeneracy property, Theorem 1.1, and some of its consequences. Next, Section 5 will be dedicated to present some applications of the main results. Blow-up analysis and some Liouville type results are proved.

\section{PRELIMINARIES}

In this section we shall present the main tools and definitions that will be used through the article. 
For $x_{0} \in \mathbb{R}^{N}$ and $r>0$, we denote by $B_{r}\left(x_{0}\right)$ the Euclidean open ball with center $x_{0}$ and radius $r$. Also, for a point $\left(x_{0}, t_{0}\right) \in \Omega \times \mathbb{R}$ and $r>0$, we consider three kinds of parabolic cylinders

$$
\begin{aligned}
& Q_{r}\left(x_{0}, t_{0}\right):=B_{r}\left(x_{0}\right) \times\left(t_{0}-r^{\theta}, t_{0}+r^{\theta}\right) \quad \text { (whole cylinder) } \\
& Q_{r}^{+}\left(x_{0}, t_{0}\right):=B_{r}\left(x_{0}\right) \times\left[t_{0}, t_{0}+r^{\theta}\right) \quad \text { (the upper semi-cylinder) } \\
& Q_{r}^{-}\left(x_{0}, t_{0}\right):=B_{r}\left(x_{0}\right) \times\left(t_{0}-r^{\theta}, t_{0}\right] \quad \text { (the lower semi-cylinder), }
\end{aligned}
$$

where $\theta$ is the intrinsic scaling factor in time variable:

$$
\theta=\theta(p, q):=\frac{p(1-q)}{p-1-q} .
$$

Moreover, we will omit the center of the cylinder as $\left(x_{0}, t_{0}\right)=(0,0)$.

For a parabolic domain $Q:=\Omega \times \mathscr{I}$, where $\mathscr{I}$ is an interval with endpoints $\mathfrak{a}<\mathfrak{b}$, we define the parabolic boundary by: $\partial_{p} Q:=(\bar{\Omega} \times\{\mathfrak{a}\}) \cup(\partial \Omega \times \mathscr{I})$.

For $p>2$ and every $\mathrm{K} \Subset \Omega_{T}$ we have according to [18, Chapter III] the following intrinsic parabolic distance:

$$
\operatorname{dist}\left(\mathrm{K}, \partial_{p} \Omega_{T}\right):=\inf _{\substack{(x, t) \in \mathrm{K} \\(y, s) \in \partial_{p} \Omega_{T}}}\left[|x-y|+\|u\|_{L^{\infty}\left(\Omega_{T}\right)}^{\frac{p-2}{p}}|t-s|^{\frac{1}{p}}\right]
$$

In the following, we give a notion of weak solution, see [18, page 11].

Definition 2.1 (Weak solution-Steklov average). A function $u \in \mathrm{V}^{1, p}\left(\Omega_{T}\right)=L^{\infty}\left((0, T) ; L^{1}(\Omega)\right) \cap L_{l o c}^{p}\left((0, T) ; W_{l o c}^{1, p}(\Omega)\right)$ is said to be a weak solution to (1.2) if, for every compact set $K \subset \Omega$ and every $0<t<T-h$ there holds:

$$
\int_{K \times\{t\}}\left[\frac{\partial u_{h}}{\partial t}+\left(|\nabla u|^{p-2} \nabla u\right)_{h} \cdot \nabla \zeta\right] d x=\int_{K \times\{t\}} f_{h}(x, u) \zeta d x
$$

for all $\zeta \in W_{0}^{1, p}(K)$, where $f_{h}(x, u)=\left(\lambda_{0} u_{+}^{q}\right)(x, t)$ and the Steklov average of a function $v$ is defined as follows:

$$
v_{h}(x, t):=\left\{\begin{array}{rll}
\frac{1}{h} \int_{t}^{t+h} v(x, \mu) d \mu & \text { if } & t \in(0, T-h] \\
0 & \text { if } \quad t \in(T-h, T) .
\end{array}\right.
$$

We point out that one takes the Steklov average since $\frac{\partial u}{\partial t}$ may not exist as a function in $\mathrm{V}^{1, p}$.

We also quote a useful comparison result for weak solutions.

Lemma 2.2 (Comparison Principle [15, Theorem 1]). Let $f \in C([0, \infty))$ be a non-negative and non-decreasing function. Assume that we have weakly:

$$
\Delta_{p} u-\frac{\partial u}{\partial t}-f(u) \leq 0 \leq \Delta_{p} v-\frac{\partial v}{\partial t}-f(v) \text { in } \quad \Omega_{T} .
$$

If $v \leq u$ in $\partial_{p} \Omega_{T}$ then $v \leq u$ in $\Omega_{T}$.

Hereafter we shall adopt the following notation:

$$
\mathscr{S}_{\left(r, x_{0}, t_{0}\right)}[u]:=\sup _{Q_{r}^{-}\left(x_{0}, t_{0}\right)} u(x, t) .
$$

Moreover, for notational simplicity, we will omit the $\left(x_{0}, t_{0}\right)$ when the center of the cylinder is the origin.

Throughout the article we shall consider the following family of solutions which are invariant under intrinsic scaling.

Definition 2.3. Let $Q_{1}$ be the unit cylinder in $\mathbb{R}^{N+1}$. For any $2 \leq p<\infty$ we say that $u \in \mathfrak{J}_{p}\left(\lambda_{0}, q\right)\left(Q_{1}\right)$ if:

$\checkmark \quad \Delta_{p} u-\frac{\partial u}{\partial t}=\lambda_{0}(x, t) u_{+}^{q}(x, t) \quad$ in $\quad Q_{1}$, for $0 \leq q<\min \{1, p-1\}=1$.

$\checkmark \quad 0 \leq u \leq 1,0<\mathfrak{m} \leq \lambda_{0} \leq \mathfrak{M}$ in $Q_{1}$.

$\checkmark \quad \frac{\partial u}{\partial t} \geq 0$ a.e. in $Q_{1}$.

$\checkmark u(0,0)=0$. 
In the next, we shall also consider for $u \in \mathfrak{J}_{p}\left(\lambda_{0}, q\right)\left(Q_{1}\right)$ the following set:

$$
\mathbb{V}_{p, q}[u]:=\left\{j \in \mathbb{N} \cup\{0\} ; \mathscr{S}_{\frac{1}{2^{j}}}[u] \leq 2^{\frac{p}{p-1-q}} \max \left\{1, \frac{1}{\mathfrak{C}_{0}^{*}}\right\} \mathscr{S}_{\frac{1}{2^{j+1}}}[u]\right\},
$$

where $\mathfrak{C}_{0}^{*}>0$ is the constant in Theorem 1.1. Moreover, observe that $\mathbb{V}_{p, q}[u]$ is not empty. Indeed, $j=0 \in \mathbb{V}_{p, q}[u]$ since, in view of Theorem 1.1:

which shows that:

$$
\mathscr{S}_{\frac{1}{2}}[u] \geq \mathfrak{C}_{0}^{*}\left(\frac{1}{2}\right)^{\frac{p}{p-1-q}} \geq \mathfrak{C}_{0}^{*}\left(\frac{1}{2}\right)^{\frac{p}{p-1-q}} \mathscr{S}_{1}[u]
$$

$$
\mathscr{S}_{1}[u] \leq 2^{\frac{p}{p-1-q}} \max \left\{1, \frac{1}{\mathfrak{C}_{0}^{*}}\right\} \mathscr{S}_{\frac{1}{2}}[u]
$$

\section{Sharp IMPROVED REgUlARITY ESTIMATES}

Through this Section we will prove an improved regularity result for weak solutions to (1.2) along their free boundaries. We begin by estimating the growth rate of functions in $\mathfrak{J}_{p}\left(\lambda_{0}, q\right)\left(Q_{1}\right)$ near the free boundary.

Lemma 3.1. There exist a positive constant $\mathfrak{C}_{0}=\mathfrak{C}_{0}(N, p, q, \mathfrak{M})$ such that:

$$
\mathscr{S}_{\frac{1}{2^{j+1}}}[u] \leq \mathfrak{C}_{0} \cdot\left(\frac{1}{2^{j}}\right)^{\frac{p}{p-1-q}}
$$

for all $u \in \mathfrak{J}_{p}\left(\lambda_{0}, q\right)\left(Q_{1}\right)$ and $j \in \mathbb{V}_{p, q}[u]$.

Proof. The proof follows the lines of [25, Lemma 2.3] adapted to our framework, and it will be achieved by contradiction. Then, let us suppose that the thesis of the lemma fails. This means that for each $k \in \mathbb{N}$ we may find $u_{k} \in \mathfrak{J}_{p}\left(\lambda_{0}, q\right)\left(Q_{1}\right)$ and $j_{k} \in \mathbb{V}_{p, q}\left[u_{k}\right]$ such that:

$$
\mathscr{S}_{\frac{1}{2^{j_{k}+1}}}\left[u_{k}\right]>k\left(\frac{1}{2^{j_{k}}}\right)^{\frac{p}{p-1-q}} .
$$

Now, for $\mathfrak{a}_{k}:=\frac{1}{2^{j_{k} p} p} \frac{1}{\frac{\mathscr{S}^{p-2}}{2^{j_{k}+1}}\left[u_{k}\right]}$ we define the auxiliary function:

$$
v_{k}(x, t):=\frac{u_{k}\left(\frac{1}{2^{j_{k}}} x, \mathfrak{a}_{k} \cdot t\right)}{\mathscr{S}_{\frac{1}{2^{j_{k}+1}}}\left[u_{k}\right]} \text { in } Q_{1}^{-} .
$$

Thus, $v_{k}$ fulfils:

$$
\begin{aligned}
& \checkmark v_{k}(0,0)=0 \text { and } \\
& \qquad \leq v_{k}(x, t) \leq \frac{\mathscr{S}_{\frac{1}{2^{j_{k}}}}\left[u_{k}\right]}{\mathscr{S}_{\frac{1}{2^{j_{k}+1}}}\left[u_{k}\right]} \leq \mathfrak{A}:=2^{\frac{p}{p-1-q}} \max \left\{1, \frac{1}{\mathfrak{C}_{0}^{*}}\right\} \text { in } Q_{1}^{-} . \\
& \left.\checkmark \quad \mathscr{S}_{\frac{1}{2}}\left[v_{k}\right] \geq 1 \text { (a consequence of }-\left(2^{-1}\right)^{\theta} \mathfrak{a}_{k} \geq-\left(2^{-j_{k}-1}\right), u_{k} \geq 0 \text { and } \frac{\partial u_{k}}{\partial t} \geq 0\right) . \\
& \checkmark \frac{\partial v_{k}}{\partial t} \geq 0 \text { a.e. in } Q_{1}^{-} . \\
& \checkmark \quad \Delta_{p} v_{k}-\frac{\partial v_{k}}{\partial t}=\frac{1}{2^{j_{k} \cdot p}} \frac{1}{\mathscr{S}^{p-1-q}\left[u_{k}\right]} \lambda_{0}\left(\frac{1}{2^{j_{k}+1}} x, \mathfrak{a}_{k} \cdot t\right)\left(v_{k}\right)_{+}^{q}(x, t) \text { in } Q_{1}^{-} .
\end{aligned}
$$


Therefore:

$$
\left\|\Delta_{p} v_{k}-\frac{\partial v_{k}}{\partial t}\right\|_{L^{\infty}\left(Q_{1}^{-}\right)}=\left\|\frac{1}{2^{j_{k} \cdot p}} \frac{1}{\frac{\mathscr{S}^{p-1-q}}{2^{j_{k}+1}}\left[u_{k}\right]} \lambda_{0}(\cdot, \cdot)\left(v_{k}\right)_{+}^{q}\right\|_{L^{\infty}\left(Q_{1}^{-}\right)} \leq \mathfrak{A}^{q} \cdot \sup _{Q_{1}} \lambda_{0}(x, t)\left(\frac{1}{k}\right)^{p-1-q} \rightarrow 0 \quad \text { as } \quad k \rightarrow \infty
$$

The previous sentences together with standard compactness arguments for parabolic $p$-Laplacian operators (cf. [18, Lemma 14.1- p.75 and Lemma 14-(iii)-p.115]) imply that, up to a subsequence, $v_{k} \rightarrow v$ locally uniformly in $\overline{Q_{\frac{4}{5}}^{-}}$. Furthermore, the limit fulfils:

$$
\begin{array}{ll}
\checkmark & \Delta_{p} v-\frac{\partial v}{\partial t}=0 \quad \text { in } \quad Q_{\frac{4}{5}}^{-} . \\
\checkmark \quad 0 \leq v \leq \mathfrak{A} \text { and } \frac{\partial v}{\partial t} \geq 0 \quad \text { a.e. in } Q_{\frac{4}{5}}^{-} . \\
\checkmark \quad \mathscr{S}_{\frac{1}{2}}[v] \geq 1 . \\
\checkmark \quad v(0, t)=0 \forall t \in\left(-\left(\frac{4}{5}\right)^{\theta}, 0\right] .
\end{array}
$$

From now on we shall analyse two possibilities:

(1) $($ Case $p=2)$

Notice that $u$ is a non-zero, non-negative caloric function in $Q_{\frac{4}{5}}^{-}$, non-decreasing in time and taking a local minimum at the origin. Hence, the strong minimum principle assures that $u$ is identically zero, which clearly yields a contradiction.

(2) (Case $p>2$ )

Due to the absence of the strong minimum principle, it will be necessary to analyse the previous limiting problem in a different manner.

Indeed, since $\frac{\partial v}{\partial t} \geq 0$, the supremum of $v$ on the 0 -level (in time) must be bigger than or equal to the ones in the corresponding negative levels. Consequently:

$$
\sup _{B_{\frac{1}{2}}} v(x, 0) \geq 1
$$

Now, we claim that $v$ is time independent, in other words, $\frac{\partial v}{\partial t} \equiv 0$ in $Q_{\frac{4}{5}}^{-}$. Indeed, if such a claim holds, then $u$ would be a non-negative $p$-harmonic function vanishing at the origin. The strong minimum principle (see [35]) says that $v \equiv 0$. However, this contradicts (3.3).

Let us prove the claim. Select $(x, t),(x, s) \in Q_{\frac{1}{2}}^{-}$and by using the property of $\mathbb{V}_{p, q}\left[u_{k}\right]$ and the $\beta$-Hölder regularity estimate (cf. [18, Theorem 1-p.41-42]):

$$
\begin{aligned}
& \left|v_{k}(x, t)-v_{k}(x, s)\right|=\frac{\left|u_{k}\left(\frac{1}{2^{j_{k}}} x, \mathfrak{a}_{k} t\right)-u_{k}\left(\frac{1}{2^{j_{k}}} x, \mathfrak{a}_{k} s\right)\right|}{\mathscr{S}_{\frac{1}{2^{j}+1}}\left[u_{k}\right]} \\
& \leq \mathfrak{A} \frac{\left|u_{k}\left(\frac{1}{2^{j_{k}}} x, \mathfrak{a}_{k} t\right)-u_{k}\left(\frac{1}{2^{j_{k}}} x, \mathfrak{a}_{k} s\right)\right|}{\mathscr{S}_{\frac{1}{2^{j_{k}}}}\left[u_{k}\right]} \\
& \leq \frac{\left.\gamma \mathfrak{A}\left\|u_{k}\right\|_{L^{\infty}\left(Q_{2}^{-} j_{k}\right.}\right)}{\mathscr{S}_{\frac{1}{2^{j} j_{k}}}\left[u_{k}\right]}\left[\frac{\mathfrak{a}_{k}\left\|u_{k}\right\|_{L^{\infty}}^{p-2}\left(Q_{2^{-j_{k}}}\right)^{|t-s|}}{\operatorname{dist}\left(Q_{2^{-\left(j_{k}+1\right)}}^{-}, \partial_{p} Q_{2^{-j_{k}}}\right)^{p}}\right]^{\frac{\beta}{p}},
\end{aligned}
$$


where $\gamma>1$ does not depend on $\left\|u_{k}\right\|_{L^{\infty}\left(Q_{2^{-j} k}^{-}\right)}$and the above intrinsic parabolic distance between sets satisfies:

$$
\begin{aligned}
& \operatorname{dist}\left(Q_{2^{-\left(j_{k}+1\right)}}^{-}, \partial_{p} Q_{2^{-j_{k}}}^{-}\right):=\inf _{\substack{\left.(x, t) \in Q_{2^{-}\left(j_{k}+1\right)}^{(}\right) \\
(y, s) \in \partial_{p} Q_{2^{-} j_{k}}}}\left[|x-y|+\left\|u_{k}\right\|_{L^{\infty}\left(Q_{2^{-j_{k}}}\right)}^{\frac{p-2}{p}}|t-s|^{\frac{1}{p}}\right] \\
& \geq\left(\frac{1}{2^{j_{k}+1}}\right)^{\frac{\theta}{p}}\left\|u_{k}\right\|_{L^{\infty}}^{\frac{p-2}{p}}\left(Q_{2^{-} j_{k}}^{-}\right)
\end{aligned}
$$

Finally:

$$
\begin{aligned}
\left|v_{k}(x, t)-v_{k}(x, s)\right| & \leq \gamma \mathfrak{A}\left[|t-s| \mathfrak{a}_{k} 2^{\left(j_{k}+1\right) \theta}\right]^{\frac{\beta}{p}} \\
& =\gamma \mathfrak{A}\left[|t-s| \frac{1}{2^{j_{k} p}} \mathscr{S}_{\frac{1}{2^{j_{k}+1}}}^{2-p}\left[u_{k}\right] 2^{\left(j_{k}+1\right) \theta}\right]^{\frac{\beta}{p}} \\
& \leq \gamma \mathfrak{A}\left[|t-s| \frac{1}{2^{j_{k} p}} k^{2-p}\left(\frac{1}{2^{j_{k}}}\right)^{\frac{p(2-p)}{p-q-1}} 2^{\left(j_{k}+1\right) \theta}\right]^{\frac{\beta}{p}} \\
& =\gamma \mathfrak{A}\left[2^{\theta} \cdot|t-s| k^{2-p}\right]^{\frac{\beta}{p}} \rightarrow 0 \text { as } k \rightarrow \infty .
\end{aligned}
$$

Therefore, $v$ is time-independent, and we finish the proof.

Remark 3.2. Notice that Lemma 3.1 assures that there exists a universal constant $0<\delta_{0} \ll 1$ (small enough) such that if $u \in \mathfrak{J}_{p}\left(\lambda_{0}, q\right)\left(Q_{1}\right)$ with:

then:

$$
\left\|\Delta_{p} u-\frac{\partial u}{\partial t}\right\|_{L^{\infty}\left(Q_{1}^{-}\right)}=\left\|\lambda_{0} u_{+}^{q}\right\|_{L^{\infty}\left(Q_{1}^{-}\right)} \leq \delta_{0}
$$

for all $j \in \mathbb{V}_{p, q}[u]$.

$$
\mathscr{S}_{\frac{1}{2^{j+1}}}[u] \leq \mathfrak{C}_{0} \cdot\left(\frac{1}{2^{j}}\right)^{\frac{p}{p-1-q}}
$$

We next state the growth property of the elements in the class $\mathfrak{J}_{p}\left(\lambda_{0}, q\right)\left(Q_{1}\right)$. Even though the proof mainly follows the guidelines of the proof of [25, Theorem 2.2], a different barrier adapted to (1.2) should be used. We quote the full proof for the convenience of the reader.

Theorem 3.3. There exists a positive constant $\mathfrak{C}=\mathfrak{C}(N, p, q, \mathfrak{M})$ such that for all $u \in \mathfrak{J}_{p}\left(\lambda_{0}, q\right)\left(Q_{1}\right)$ there holds:

$$
u(x, t) \leq \mathfrak{C} . \mathfrak{d}(x, t)^{\frac{p}{p-1-q}} \quad \forall(x, t) \in Q_{\frac{1}{2}},
$$

where:

$$
\mathfrak{d}(x, t):=\left\{\begin{array}{cc}
\sup \left\{r \geq 0 ; Q_{r}(x, t) \subset\{u>0\}\right\} & \text { for } \\
0 & \text { otherwise. }
\end{array}(x, t) \in\{u>0\}\right.
$$

Proof. The proof will be achieved by an inductive reasoning. First of all, we claim that:

$$
\mathscr{S}_{\frac{1}{2^{j}}}[u] \leq \mathfrak{C}_{0}\left(\frac{1}{2^{j-1}}\right)^{\frac{p}{p-1-q}} \quad \forall j \in \mathbb{N},
$$

where $\mathfrak{C}_{0}$ is the constant coming from Lemma 3.1. Note that if $\mathfrak{C}_{0} \geq 1$, which we can suppose without loss of generality, then (3.4) holds for $j=0$. Suppose now that (3.4) holds for some $j \in \mathbb{N}$. We will verify the $(j+1)^{\text {th }}$ step of induction. In fact, if $j \in \mathbb{V}_{p, q}[u]$ then the result holds directly by Lemma 3.1. On the other hand, if (3.4) fails, then we obtain by using the inductive hypothesis, the following estimates: 


$$
\mathscr{S}_{\frac{1}{2^{j+1}}}[u] \leq\left(\frac{1}{2}\right)^{\frac{p}{p-1-q}} \cdot \mathscr{S}_{\frac{1}{2^{j}}}[u] \leq \mathfrak{C}_{0} \cdot\left(\frac{1}{2}\right)^{\frac{p}{p-1-q}}\left(\frac{1}{2^{j-1}}\right)^{\frac{p}{p-1-q}}=\mathfrak{C}_{1} \cdot\left(\frac{1}{2^{j}}\right)^{\frac{p}{p-1-q}} .
$$

Therefore, (3.4) holds for all $j \in \mathbb{N}$.

Now, in order to finish the proof for a continuous parameter $r \in(0,1)$ let $j \in \mathbb{N}$ be the greatest integer such that $\frac{1}{2^{j+1}} \leq r<\frac{1}{2^{j}}$. Then:

$$
\mathscr{S}_{r}[u] \leq \mathscr{S}_{\frac{1}{2^{j}}}[u] \leq \mathfrak{C}_{0} \cdot\left(\frac{1}{2^{j-1}}\right)^{\frac{p}{p-1-q}} \leq \mathfrak{C}(N, p, q, \mathfrak{M}) \cdot r^{\frac{p}{p-1-q}} .
$$

Finally, in order to obtain an estimate for $u$ over the whole cylinder we will use a suitable barrier function from above. Let:

where the first constant:

$$
\Phi(x, t)=\mathfrak{c}_{1} \cdot\left(\mathfrak{a} \cdot|x|^{\frac{p}{p-1}}+\mathfrak{b} \cdot t^{\frac{p-1-q}{(p-1)(1-q)}}\right)^{\frac{p-1}{p-1-q}}
$$

$$
\mathfrak{c}_{1}:=\left(\frac{\mathfrak{m}}{\left(N+\frac{p q}{p-1-q}\right)}\left(\frac{p-1-q}{\mathfrak{a} \cdot p}\right)^{p-1}\right)^{\frac{1}{p-1-q}}
$$

is chosen so that:

$$
\Delta_{p} \Phi-\frac{\partial \Phi}{\partial t}-\lambda_{0}(x, t) . \Phi^{q} \leq 0=\Delta_{p} u-\frac{\partial u}{\partial t}-\lambda_{0}(x, t) u_{+}^{q}
$$

in $Q_{1}^{+}$, for all $\mathfrak{a}, \mathfrak{b}>0$. Finally, pick $\mathfrak{b}>\mathfrak{a}$ with $\mathfrak{a}$ large enough so that $\Phi \geq u$ on $\partial_{p} Q_{1}^{+}$, where we have used that $\mathscr{S}_{r}[u] \leq \mathfrak{c} \cdot r^{\frac{p}{p-1-q}}$ for the estimate on $\{t=0\}$. Consequently, the comparison principle (Lemma 2.2) implies that $\Phi \geq u$ in $Q_{1}^{+}$. Therefore:

$$
\sup _{Q_{r}} u(x, t) \leq \mathfrak{C}(N, p, q, \mathfrak{m}) \cdot r^{\frac{p}{p-1-q}}
$$

We now are ready to prove the main result of the article.

Proof of Theorem 1.2. In order to prove Theorem 1.2, we have to reduce the hypothesis presented on it to the framework of Theorem 3.3. We assume without loss of generality that $\mathrm{K}=\overline{Q_{1}} \subset \Omega_{T}$. For $(x, t) \in\{u>0\} \cap \mathrm{K}$ let $\mathfrak{d}(x, t)$ the distance coming from Theorem 3.3. For $\left(x_{0}, t_{0}\right) \in \partial\{u>0\} \cap \mathrm{K}$ let us define:

$$
v(y, s):=\frac{u\left(x_{0}+\mathfrak{R}_{0} y, t_{0}+\mathfrak{R}_{0}^{\theta} s\right)}{\kappa_{0}} \quad \text { in } Q_{1}
$$

for $\kappa_{0}, \mathfrak{R}_{0}>0$ constants to be determined a posteriori. From the equation satisfied by $u$, we easily verify that $v$ fulfils in the weak sense:

$$
\Delta_{p} v-\frac{\partial v}{\partial t}=\hat{\lambda}_{0}(x, t) \cdot v_{+}^{q}(y, s)
$$

for an appropriate bounded function $\hat{\lambda}_{0}(x, t)$. Now, let $\delta_{\lambda_{0}, p, q}=\delta_{0}>0$ be the greatest universal constant, granted by the Remark 3.2 such the Lemma 3.1 holds provided:

$$
\left\|\Delta_{p} v-\frac{\partial v}{\partial t}\right\|_{L^{\infty}\left(Q_{1}\right)}=\left\|\hat{\lambda_{0} v_{+}^{q}}\right\|_{L^{\infty}\left(Q_{1}\right)} \leq \delta_{\lambda_{0}, p, q} .
$$


Then, we make the following choices in the definition of $v$ :

$$
\kappa_{0}:=\|u\|_{L^{\infty}\left(\Omega_{T}\right)} \quad \text { and } \quad \mathfrak{R}_{0}:=\min \left\{1, \frac{\operatorname{dist}\left(\mathrm{K}, \partial_{p} \Omega_{T}\right)}{2}, \sqrt[p]{\kappa_{0}}, \sqrt[p]{\frac{\delta_{0} \cdot \kappa_{0}^{p-1-q}}{\mathfrak{M}}}\right\} .
$$

Finally, with such selections $v$ fits into the framework of Theorem 3.3.

Remark 3.4. In view of previous results, we must highlight the relationship between regularity coming from deadcore solutions and the one coming from the classical Schauder theory. For this end, let us suppose that $u$ is a classical solution to

$$
\Delta u-\frac{\partial u}{\partial t}=\lambda_{0}(x, t) u_{+}^{q}(x, t) \quad \text { in } \quad Q_{1},
$$

where $0<q<1$ and $\lambda_{0} \in C^{0, q}\left(Q_{1}\right)$. Thus, under such assumptions the Schauder theory assures that solutions to (3.7) are $C_{\mathrm{loc}}^{2+q, \frac{2+q}{2}}\left(Q_{1}\right)$ (particularly at free boundary points). On the other hand, our main Theorem 3.3 claims that $u$ is $C^{\kappa+\alpha, \frac{\kappa+\alpha}{2}}$ at free boundary points, where

$$
\kappa:=\left\lfloor\frac{2}{1-q}\right\rfloor \quad \text { and } \quad \alpha:=\frac{2}{1-q}-\left\lfloor\frac{2}{1-q}\right\rfloor .
$$

Nevertheless, notice that for any $0<q<1$ one has

$$
\frac{2}{1-q}>2+q
$$

which means that dead-core solutions are more regular, along free boundary points, that the best regularity result coming from classical regularity theory (compare with [10] for improved regularity estimates in the context of flat solutions and [12, Theorem 1.1] for similar estimates in the context of fully nonlinear parabolic dead core problems).

Next, we shall analyse the regularity behaviour for weak solutions to the critical case $q=p-1$ :

$$
\Delta_{p} u-\frac{\partial u}{\partial t}=\lambda_{0}(x, t) \cdot u_{+}^{p-1}(x, t) \quad \text { in } \quad \Omega_{T},
$$

where $0<\mathfrak{m} \leq \lambda_{0} \leq \mathfrak{M}$ and $1<p<\infty$.

An interesting issue in this context is the following: Does the critical case permit the formation of dead-core regions? By means of an energy argument, we shall prove that a non-negative solution to (3.8) cannot vanish at interior points, unless it is identically zero.

Theorem 3.5 (Strong Maximum Principle). Let $u$ be a non-negative weak solution to (3.8) for $2 \leq p<\infty$. If there exists a point $\left(x_{0}, t_{0}\right) \in \Omega_{T}$ such that $u\left(x_{0}, t_{0}\right)=0$, then $u \equiv 0$ in $\Omega_{T}$.

Proof. From standard energy estimates we have:

$$
\frac{1}{2} \frac{d}{d t} \int_{\Omega}|u(x, t)|^{2} d x+\int_{\Omega}|\nabla u|^{p} d x=\int_{\Omega} \lambda_{0}(x, t)|u|^{p} d x \leq \mathfrak{M} \int_{\Omega}|u|^{p} d x .
$$

Moreover, by using the variational characterization of $\mathfrak{M}$, for example, via Poincaré's inequality we obtain:

$$
\frac{1}{2} \frac{d}{d t} \int_{\Omega}|u(x, t)|^{2} d x \leq-\int_{\Omega}|\nabla u|^{p} d x+\mathfrak{M} \int_{\Omega}|u|^{p} d x \leq 0 .
$$

Finally, we conclude that $u(x, t)=0$ in $\Omega_{T}$, since $u\left(x_{0}, t_{0}\right)=0$. 
Example 3.6. Theorem 3.5 assures that non-trivial weak solutions must be strictly positive when $q=p-1$. As a matter of fact, fixed any direction $i=1, \cdots, N$ and a constant $\lambda_{0}>0$. We then have that:

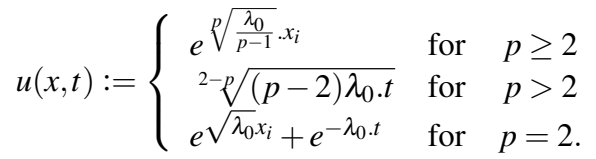

is a smooth and strictly positive solutions to:

$$
\Delta_{p} u(x, t)-\frac{\partial u}{\partial t}(x, t)=\lambda_{0} \cdot u^{p-1}(x, t) \quad \text { in } \quad \Omega_{T} .
$$

Finally, using Lemma 3.1 we are able to prove a similar growth rate for the gradient of a particular family of functions.

In order to prove the gradient estimate we must restrict ourselves to the following class of functions:

Definition 3.7. We say that $v \in \mathfrak{R}_{p}^{\infty}\left(Q_{1}\right)$ provided:

(1) $v$ is a weak solution to:

$$
\Delta_{p} v-\frac{\partial v}{\partial t}=f \in L^{\infty}\left(Q_{1}\right)
$$

and fulfils the following a priori estimate:

$$
\mathscr{S}_{\frac{1}{2}}[|\nabla v(x, t)|] \leq \mathfrak{C}(N, p)\left[\|v\|_{L^{\infty}\left(Q_{1}\right)}+\|f\|_{L^{\infty}\left(Q_{1}\right)}^{\frac{1}{p-1}}\right] .
$$

(2) $\mathscr{S}_{1}[|\nabla v(x, t)|] \leq 1$.

(3) For each $j \in \mathbb{N}$ there exists a universal constant $\kappa_{j}>0$ with $\liminf _{k \rightarrow \infty} \kappa_{j}>0$ such that:

$$
\begin{aligned}
& \sup ^{B \frac{1}{2^{j+1}} \times\left(-\left(\frac{1}{2}\right)^{\theta} \alpha_{j}, 0\right)}|\nabla v(x, t)| \geq \kappa_{j} \mathscr{S}_{\frac{1}{2^{j+1}}}[|\nabla v(x, t)|] \quad \forall j \in \mathbb{N}, \\
& \text { where } \alpha_{j}:=\frac{1}{2^{2 j}} \cdot \frac{1}{\mathscr{S}^{p-2} \frac{1}{2^{j+1}}[|\nabla v(x, t)|]} .
\end{aligned}
$$

Observe that for $p=2$ the class $\mathfrak{R}_{p}^{\infty}\left(Q_{1}\right) \neq \emptyset$. Moreover, in this case $\kappa_{j}=1$ for all $j \in \mathbb{N}$ (compare with [10, Section 2.3], [12, Corollary 4.1], [14, Section 5 and 6] and the references therein).

Lemma 3.8. If $\mathfrak{R}_{p}^{\infty}\left(Q_{1}\right)$ is not empty. Then, there exists a positive constant $\mathfrak{C}_{1}=\mathfrak{C}_{1}(N, p, q, \mathfrak{M})$ such that for all $u \in \mathfrak{J}_{p}\left(\lambda_{0}, q\right)\left(Q_{1}\right) \cap \mathfrak{R}_{p}^{\infty}\left(Q_{1}\right)$ there holds:

$$
|\nabla u(x, t)| \leq \mathfrak{C}_{1} \cdot \mathfrak{d}(x, t)^{\frac{1+q}{p-1-q}} \quad \forall(x, t) \in Q_{\frac{1}{2}},
$$

Proof. As before, it is enough to prove the following estimate:

$$
\mathscr{S}_{\frac{1}{2^{j+1}}}[|\nabla u(x, t)|] \leq \max \left\{\mathfrak{C}_{2} \cdot\left(\frac{1}{2^{j}}\right)^{\frac{1+q}{p-1-q}},\left(\frac{1}{2}\right)^{\frac{1+q}{p-1-q}} \mathscr{S}_{\frac{1}{2^{j}}}[|\nabla u(x, t)|]\right\},
$$

for all $j \in \mathbb{N}$ and a constant $\mathfrak{C}_{2}=\mathfrak{C}_{2}(N, p, q, \mathfrak{M})$.

Let us suppose that (3.9) does not hold. Then, there exists $u_{j} \in \mathfrak{J}_{p}\left(\lambda_{0}, q\right)\left(Q_{1}\right) \cap \mathfrak{R}_{p}^{\infty}\left(Q_{1}\right)$ such that:

$$
\mathscr{S}_{\frac{1}{2^{j+1}}}\left[\left|\nabla u_{j}(x, t)\right|\right] \geq \max \left\{j\left(\frac{1}{2^{j}}\right)^{\frac{1+q}{p-1-q}},\left(\frac{1}{2}\right)^{\frac{1+q}{p-1-q}} \mathscr{S}_{\frac{1}{2^{j}}}\left[\left|\nabla u_{j}(x, t)\right|\right]\right\} .
$$


Next, we define the auxiliary normalized and scaled function:

$$
v_{j}(x, t):=\frac{2^{j} u_{j}\left(\frac{1}{2^{j}} x, \alpha_{j} t\right)}{\mathscr{S}_{\frac{1}{2^{j+1}}}\left[\left|\nabla u_{j}(x, t)\right|\right]}
$$

Notice that due to (3.10) we get:

$$
\alpha_{j}<\left(\frac{1}{j}\right)^{p-1-q} \cdot \frac{1}{2^{j(1-q)}} \rightarrow 0 \quad \text { as } \quad j \rightarrow \infty .
$$

Next, using (3.5) and (3.10) we obtain:

$$
0 \leq v_{j}(x, t) \leq \frac{2^{j} \mathfrak{C}\left(2^{-j}\right)^{\frac{p}{p-1-q}}}{\mathscr{S}_{\frac{1}{2^{j+1}}}\left[\left|\nabla u_{j}(x, t)\right|\right]} \leq \frac{\mathfrak{C}}{j} \quad \text { for } \quad(x, t) \in Q_{1}
$$

Furthermore,

$$
\begin{gathered}
\checkmark \Delta_{p} v_{j}-\frac{\partial v_{j}}{\partial t}=\hat{\lambda_{0}}(x, t)\left(v_{j}\right)_{+}^{q}(x, t) \quad \text { in } \quad Q_{1} \text { in the weak sense, where } \\
\hat{\lambda}_{0}(x, t):=\frac{1}{2^{j(1+q)}} \frac{1}{\mathscr{S}^{p-1-q}\left[\left|\nabla u_{j}(x, t)\right|\right]} \lambda_{0}\left(\frac{1}{2^{j}} x, \alpha_{j} . t\right) . \\
\checkmark \mathscr{S}_{\frac{1}{2}}\left[\left|\nabla v_{j}(x, t)\right|\right] \geq \kappa_{j}>0 \text { due to } v_{j} \in \mathfrak{R}_{p}^{\infty}\left(Q_{1}\right) .
\end{gathered}
$$

Consequently, we have that:

$$
\left\|\hat{\lambda_{0}} \cdot\left(v_{j}\right)_{+}^{q}\right\|_{L^{\infty}\left(Q_{1}\right)} \leq \mathfrak{M} \cdot \mathfrak{C}^{q} \cdot\left(\frac{1}{j}\right)^{p-1} .
$$

Finally, by using the a priori gradient estimate from $\mathfrak{R}_{p}^{\infty}\left(Q_{1}\right)$ we obtain:

$$
\kappa_{j} \leq \mathscr{S}_{\frac{1}{2}}\left[\left|\nabla v_{j}(x, t)\right|\right] \leq \mathfrak{C}(N, p)\left[\left\|v_{j}\right\|_{L^{\infty}\left(Q_{1}\right)}+\left\|\hat{\lambda_{0}}\left(v_{j}\right)_{+}^{q}\right\|_{L^{\infty}\left(Q_{1}\right)}^{\frac{1}{p-1}}\right] \leq \mathfrak{C}^{*} \cdot \frac{1}{j} \rightarrow 0 \quad \text { as } \quad j \rightarrow \infty,
$$

which is a contradiction. Therefore the proof is ended.

\section{NON-DEGENERACY AND ITS CONSEQUENCES}

Throughout this section we will deliver the proof of Non-degeneracy property for weak solutions. Thereafter, we will present important consequences of this fundamental property.

Proof of Theorem 1.1. Notice that by continuity of weak solutions it suffices to show the thesis for points in $\{u>0\}$. Fix $\left(x_{0}, t_{0}\right) \in\{u>0\}$. Let $r>0$ be so that $Q_{r}\left(x_{0}, t_{0}\right) \subset Q_{1}$, and consider the scaled functions:

Observe that:

$$
u_{r}(x, t)=\frac{u\left(x_{0}+r x, t_{0}+r^{\theta} t\right)}{r^{\overline{p-1-q}}}, \quad(x, t) \in \overline{Q_{1}^{-}} .
$$

$$
\Delta_{p} u_{r}-\frac{\partial u_{r}}{\partial t}-\lambda_{0}\left(x_{0}+r x, t_{0}+r^{\theta} t\right) u_{r}^{q}=0 \quad \text { in } Q_{1}^{-} .
$$

We introduce a comparison function defined in $\overline{Q_{1}^{-}}$:

$$
\hat{\Phi}(x, t):=\mathfrak{c} \cdot\left(|x|^{\frac{p}{p-1}}+(-t)^{\frac{p-1-q}{(p-1)(1-q)}}\right)^{\frac{p-1}{p-1-q}},
$$

where

$$
\mathfrak{c}=\min \left\{\left[\frac{\mathfrak{m}(1-q)}{2}\right]^{\frac{1}{1-q}},\left[\frac{\mathfrak{m}}{2}\left(\frac{p-1-q}{p}\right)^{p-1}\left(N+\frac{p q}{p-1-q}\right)\right]^{\frac{1}{p-1-q}}\right\}
$$


It follows from (4.1) that:

$$
\Delta_{p} \hat{\Phi}-\frac{\partial \hat{\Phi}}{\partial t}-\lambda_{0}(x, t) . \hat{\Phi}^{q} \leq 0 .
$$

Now, if $u_{r} \leq \hat{\Phi}$ on the parabolic boundary of $Q_{1}^{-}$, then Lemma 2.2 would imply $u_{r} \leq \hat{\Phi}$ in $Q_{1}^{-}$. But this contradicts $\hat{\Phi}(0,0)=0<u_{r}(0,0)$. Hence, there should be a point $\left(x^{\prime}, t^{\prime}\right)$ on $Q_{1}^{-}$so that:

$$
u_{r}\left(x^{\prime}, t^{\prime}\right) \geq \hat{\Phi}\left(x^{\prime}, t^{\prime}\right) \text {. }
$$

Scaling back proves the result.

Such a Non-degeneracy property and the growth rate for weak solutions to (1.2) will drive us to establish some measure-theoretical properties of the free boundary. We start by showing a property of positive density.

Corollary 4.1 (Positive Lebesgue density of $\{u>0\}$ ). Let $u$ be the weak solution to (1.2). Then, there exists a positive constant $\rho=\rho\left(N, p, \lambda_{0},\|u\|_{L^{\infty}\left(Q_{1}\right)}\right)$ such that for all $\left(x_{0}, t_{0}\right) \in \overline{\{u>0\}}$ and $0<r<1$ so that $Q_{r}\left(x_{0}, t_{0}\right) \subset$ $Q_{\frac{1}{2}}$, the inclusion:

$$
Q_{\rho r}\left(x^{\prime}, t^{\prime}\right) \subset Q_{r}\left(x_{0}, t_{0}\right) \cap\{u>0\},
$$

holds for some $\left(x^{\prime}, t^{\prime}\right) \in Q_{r}^{-}\left(x_{0}, t_{0}\right)$.

Proof. Let $\left(x_{0}, t_{0}\right) \in \overline{\{u>0\}} \cap \overline{Q_{1}}$. For $r$ small enough, we have by Theorem 1.1 that there exists $\left(x^{\prime}, t^{\prime}\right) \in$ $Q_{\frac{r}{2}}^{-}\left(x_{0}, t_{0}\right)$ such that:

$$
u\left(x^{\prime}, t^{\prime}\right) \geq \mathfrak{C}_{0}^{*} \cdot\left(\frac{r}{2}\right)^{\frac{p}{p-q-1}} .
$$

Suppose that for all $0<\mathfrak{d}<1$ small, there exists a point $(x, t) \in \partial\{u>0\} \cap \overline{Q_{\frac{1}{2}}}$ satisfying:

$$
\left(x^{\prime}, t^{\prime}\right) \in Q_{\mathfrak{d}}(x, t) \subset Q_{r}\left(x_{0}, t_{0}\right) .
$$

Now, according to (4.3), (4.4) and Theorem 3.3, it follows:

$$
\mathfrak{C}_{0}^{*} \cdot\left(\frac{r}{2}\right)^{\frac{p}{p-q-1}} \leq u\left(x^{\prime}, t^{\prime}\right) \leq \sup _{Q_{\mathfrak{d}}(x, t)} u \leq \mathfrak{C}, \mathfrak{d}^{\frac{p}{p-q-1}} .
$$

This clearly does not hold for $\mathfrak{d}<\imath \cdot \frac{r}{2}$, where:

$$
\imath:=\left(\frac{\mathfrak{C}_{0}^{*}}{\mathfrak{C}^{\mathfrak{C}}}\right)^{\frac{p-q-1}{p}}<1
$$

Hence

$$
Q_{\frac{l}{4} r}\left(x^{\prime}, t^{\prime}\right) \subset Q_{r}\left(x_{0}, t_{0}\right) \cap\{u>0\}
$$

This ends the proof of the theorem.

Remark 4.2. Notice that Corollary 4.1 assures that the free boundary cannot have Lebesgue points. Consequently, for any compact set $\mathrm{K} \subset Q_{1}$, we have:

$$
\mathcal{L}^{N+1}(\partial\{u>0\} \cap \mathrm{K})=0 .
$$

Next, we shall prove, as an easy consequence of the above result, that the free boundary is a porous set. We recall the definition of this notion.

Definition 4.3 (Porous set). A set $\mathscr{E} \in \mathbb{R}^{N}$ is said to be porous with porosity constant $0<\varsigma \leq 1$ if there exists $\mathfrak{R}>0$ such that for each $x_{0} \in \mathscr{E}$ and $0<\mathfrak{r}<\mathfrak{R}$ there is a point $x^{\prime}$ so that $B_{\mathfrak{r} \varsigma}\left(x^{\prime}\right) \subset B_{\mathfrak{r}}\left(x_{0}\right) \backslash \mathscr{E}$.

Observe that a porous set has Hausdorff dimension at most $N-c_{0} \varsigma^{N}$, where $c_{0}=c_{0}(N)>0$. In particular, a porous set has Lebesgue measure zero (cf. [36]). 
Corollary 4.4 (Porosity for $t$-level of free boundary). Let $u$ be the weak solution to (1.2). For every compact set $\mathrm{K} \subset Q_{1}$ holds that:

$$
\mathscr{H}^{N-\delta}\left(\partial\{u>0\} \cap \mathrm{K} \cap\left\{t=t_{0}\right\}\right)<\infty
$$

for a constant $0<\delta=\delta\left(N, p, q, \lambda_{0},\|u\|_{L^{\infty}\left(Q_{1}\right)}, \operatorname{dist}\left(\mathrm{K}, \partial_{p} Q_{1}\right)\right) \leq 1$.

Proof. Without loss of generality we can suppose that $\mathrm{K}=\overline{Q_{\frac{1}{2}}}$. Let $\left(z, t_{0}\right) \in \partial\{u>0\} \cap \overline{Q_{\frac{1}{2}}}$ then for $0<r \ll 1$, according to Non-degeneracy property, there exists $x^{\prime} \in \partial B_{r}(z)$ such that:

$$
u\left(x^{\prime}, t_{0}\right) \geq \mathfrak{C}_{0}^{*} \cdot r^{\frac{p}{p-1-q}} .
$$

On the other hand, from Theorem 3.3:

$$
u\left(x^{\prime}, t_{0}\right) \leq \mathfrak{C} \cdot \mathfrak{d}\left(x^{\prime}, t_{0}\right)^{\frac{p}{p-1-q}}
$$

Consequently:

$$
\mathfrak{C}_{0}^{*} \cdot r^{\frac{p}{p-1-q}} \leq u\left(x^{\prime}, t_{0}\right) \leq \mathfrak{C} \cdot \mathfrak{d}\left(x^{\prime}, t_{0}\right)^{\frac{p}{p-1-q}} .
$$

Next, by selecting $\delta^{*}=\left(\frac{\mathfrak{C}_{0}^{*}}{\mathfrak{C}}\right)^{\frac{p-1-q}{p}}$, then $\mathfrak{d}\left(x^{\prime}, t_{0}\right) \geq \delta^{*} r$ for a $0<\delta^{*} \leq 1$. Therefore:

$$
B_{\delta^{*} r}\left(x^{\prime}\right) \cap B_{r}(z) \subset\{u>0\} \cap \Omega_{T} .
$$

Now, choose $y \in\left[z, x^{\prime}\right]$ such that $\left|y-x^{\prime}\right|=\frac{\delta^{*} r}{2}$. Note that for any $y_{0} \in B_{\frac{\delta^{*} r}{2}}(y)$ we have:

$$
\left|y_{0}-x^{\prime}\right| \leq\left|y_{0}-y\right|+\left|y-x^{\prime}\right|=\delta^{*} r .
$$

Moreover, since $\left|z-x^{\prime}\right|=|y-z|+\left|y-x^{\prime}\right|$ then:

$$
\left|y_{0}-z\right| \leq\left|y_{0}-y\right|+\left(\left|z-x^{\prime}\right|-\left|y-x^{\prime}\right|\right) \leq \frac{\delta^{*} r}{2}+\left(r-\frac{\delta^{*} r}{2}\right)=r
$$

so, we conclude that:

$$
B_{\frac{\delta^{*} r}{2}}(y) \subset B_{\delta^{*} r}\left(x^{\prime}\right) \cap B_{r}(z) \subset B_{r}(z) \backslash \partial\{u>0\} .
$$

Therefore, $\partial\{u>0\} \cap\left\{t=t_{0}\right\} \cap \mathrm{K}$ is porous with porosity constant $\delta:=\frac{\delta^{*}}{2}$.

In contrast to one of the most known properties of heat equation, namely the infinity speed of propagation, parabolic $p$-dead-core solutions have the property of finite speed propagation. Such a property supports the physical soundness of the equation to diffusive models. Moreover, the occurrence of this phenomenon is consequence of the degeneracy of the equation at the level set $u=0$. Such a property is well-known in the literature and can be obtained by several different methods (cf. Díaz [15, Theorem 10] for an alternative approach). The next result is similar to the one in [9, Corollary 4.4]. Hence, we will only write the modifications for the reader's convenience.

Corollary 4.5 (Finite speed propagation of $\{u>0\}$ ). There exists a constant $\mathfrak{c}(N, p, q) \geq 1$ such that, for any solution to (1.2), with non-negative and bounded time derivative, and any $Q_{r}^{+}\left(x_{0}, t_{0}\right) \subset \Omega_{T}$, the implication:

$$
u\left(\cdot, t_{0}\right)=0 \quad \text { in } \quad B_{r}\left(x_{0}\right) \Rightarrow u\left(\cdot, t_{0}+\mathfrak{s}^{\theta}\right)=0 \quad \text { in } \quad B_{\max \{0, r-\mathfrak{c s}\}}\left(x_{0}\right)
$$

holds.

Proof. Let us suppose for sake of contradiction that for $0<\mathfrak{s}_{1}<\frac{r}{\mathfrak{c}}$ there exists a point $x_{1} \in B_{r-\mathfrak{c s}_{1}}\left(x_{0}\right)$ such that $u\left(x_{1}, t_{0}+\mathfrak{s}_{1}^{\theta}\right)>0$. The Non-degeneracy property (Theorem 1.1) implies that:

$$
u\left(x_{2}, \varsigma\right) \geq \mathfrak{C}_{0}^{*} \mathfrak{s}_{1}^{\frac{p}{p-1-q}}
$$

for some $\left(x_{2}, \varsigma\right) \in \overline{Q_{\mathfrak{s}_{1}}^{-}\left(x_{1}, t_{0}+\mathfrak{s}_{1}^{\theta}\right)}$. Moreover, since $\frac{\partial u}{\partial t}$ is non-negative and bounded we deduce that there exists $0<\tau(n, p, q)<1$ and $\left(x_{2}, t_{0}+\mathfrak{s}_{2}^{\theta}\right)$ satisfying:

$$
u\left(x_{2}, t_{0}+\mathfrak{s}_{2}^{\theta}\right)>0, \quad \text { with } \quad 0 \leq \mathfrak{s}_{2} \leq(1-\tau) \mathfrak{s}_{1} \quad \text { and } \quad\left|x_{2}-x_{1}\right| \leq \mathfrak{s}_{1} .
$$


By iterating the previous reasoning we can obtain a point $\left(x_{k}, t_{0}+\mathfrak{s}_{k}^{\theta}\right)$ so that:

$$
u\left(x_{k}, t_{0}+\mathfrak{s}_{k}^{\theta}\right)>0, \quad \text { with } \quad 0 \leq \mathfrak{s}_{k} \leq(1-\tau)^{k-1} \mathfrak{s}_{1} \quad \text { and } \quad\left|x_{k}-x_{1}\right| \leq \frac{\mathfrak{s}_{1}\left[1-(1-\tau)^{k-1}\right]}{\tau} .
$$

Finally, up to a subsequence $x_{k} \rightarrow x_{\infty}$ as $k \rightarrow \infty$, thus we obtain a point $\left(x_{\infty}, t_{0}\right) \in \overline{\{u>0\}}$ fulfilling $\left|x_{\infty}-x_{1}\right|<\frac{\mathfrak{s}_{1}}{\tau}$. However, this contradicts our assumptions provided $\mathfrak{c} \geq \frac{4}{\tau}$. This contradiction proves the corollary.

\section{Global anAlysis RESUlts}

Blow-up analysis. Throughout this Section we shall study the blow-up analysis over free boundary points (interior touching points). Thus, let $u$ be a solution to (1.2) and $\left(x_{0}, t_{0}\right) \in \partial\{u>0\} \cap Q_{\frac{1}{2}}$. Now, consider, for each $\varepsilon>0$, the blow-up family $u_{\varepsilon}: Q_{\frac{1}{2}} \rightarrow \mathbb{R}$ given by:

$$
u_{\varepsilon}(x, t):=\frac{u\left(x_{0}+\varepsilon x, t_{0}+\varepsilon^{\theta} t\right)}{\varepsilon^{\frac{p}{p-q-1}}} .
$$

We must stress that this sequence is indeed an $\varepsilon$-zoom-in of $u$ re-scaled in a suitable way. Let us analyse the "limiting profiles". Note that $u_{\varepsilon}$ fulfils in the weak sense:

$$
\Delta_{p} u_{\varepsilon}-\frac{\partial u_{\varepsilon}}{\partial t}=\lambda_{0}\left(x_{0}+\varepsilon x, t_{0}+\varepsilon^{\theta} t\right) \cdot\left(u_{\varepsilon}\right)_{+}^{q} \quad \text { in } \quad Q_{\frac{1}{2 \varepsilon}} .
$$

From Theorem 3.3 we have that:

$$
u_{\varepsilon}(x, t) \leq C\left(N, p, q, \lambda_{0}\right) \quad \forall(x, t) \in Q_{\frac{1}{2 \varepsilon}} .
$$

Particularly, $u_{\varepsilon}$ is locally bounded in $Q_{\frac{1}{2 \varepsilon}}$. From universal Hölder regularity, see for instance [18], [20] and [34], up to a subsequence $u_{\varepsilon} \rightarrow u_{0}$ locally uniformly to an entire function.

From now on, $u_{0}$ will denote a limiting function coming from the previous reasoning. For this reason, we will label it as Blow-up solution at $\left(x_{0}, t_{0}\right) \in \partial\{u>0\}$. The next Theorem 5.1 establishes a quantitative control profile at infinity for a class of entire solutions to the dead-core problem, namely blow-up solutions (compare with [31, Section 3.1]).

Theorem 5.1 (Behaviour of Blow-up solutions). Let $u_{0}$ be a Blow-up solution at $\left(x_{0}, t_{0}\right) \in \partial\{u>0\}$. Then $u_{0}(0,0)=0$ and

$$
\Delta_{p} u_{0}-\frac{\partial u_{0}}{\partial t}=\lambda_{0}(0,0) \cdot\left(u_{0}\right)_{+}^{q}(x, t) \quad \text { in } \quad \mathbb{R}^{N} \times \mathbb{R}
$$

in the weak sense. Moreover, there exist universal constants $c_{0}, C_{0}>0$ such that:

$$
c_{0} \leq \liminf _{|x|+|t|^{\frac{1}{\theta}} \rightarrow \infty} \frac{u_{0}(x, t)}{\left(|x|+|t|^{\frac{1}{\theta}}\right)^{\frac{p}{p-1-q}}} \leq \limsup _{|x|+|t|^{\frac{1}{\theta}} \rightarrow \infty} \frac{u_{0}(x, t)}{\left(|x|+|t|^{\frac{1}{\theta}}\right)^{\frac{p}{p-1-q}}} \leq C_{0} .
$$

Proof. Since each $u_{\varepsilon}$ fulfils $u_{\varepsilon}(0,0)=0$ and:

$$
\Delta_{p} u_{\varepsilon}-\frac{\partial u_{\varepsilon}}{\partial t}=\lambda_{0} \cdot\left(u_{\varepsilon}\right)_{+}^{q}
$$

in the weak sense, we can finish the proof by using stability results (compactness) as in the proof of Lemma 3.1 (see also [18], [20] and [34]). Finally, the lower and upper control at infinity come from Theorems 3.3 and Theorem 1.1 .

Remark 5.2. Note that Blow-up solutions are non-trivial. Moreover, such solutions fulfils:

$$
c_{0} \cdot r^{\frac{p}{p-q-1}} \leq \mathscr{S}_{r}\left[u_{0}\right] \leq C_{0} \cdot r^{\frac{p}{p-q-1}},
$$

for values of $r$ large enough. 
Remark 5.3. In view of Theorem 5.1 the non-trivial space-independent blow up solution $u=u(t)$ to:

$$
\Delta_{p} u-\frac{\partial u}{\partial t}=\lambda_{0} u_{+}^{q}
$$

is given by:

$$
u(t)=\left[(1-q) \lambda_{0}\left(t_{0}-t\right)\right]_{+}^{\frac{1}{1-q}} .
$$

On the other hand, non-trivial time-independent blow up solutions $u=u(x)$ are of the following form:

$$
u(x)=\left\{C_{p, q} \cdot\left(x_{i}\right)_{+}^{\frac{p}{p-1-q}}, C_{p, q} \cdot\left(x_{i}\right)_{-}^{\frac{p}{p-1-q}}, C_{p, q} \cdot\left(\left|x-x_{0}\right|-\mathfrak{R}_{0}\right)_{+}^{\frac{p}{p-1-q}}\right\}
$$

for any $i=1, \cdots, N$, where:

$$
C_{p, q}:=\left(\lambda_{0} \cdot \frac{(p-q-1)^{p}}{p^{p-1}(p q+N(p-1-q))}\right)^{\frac{1}{p-q-1}} \cdot( \pm x)_{+}^{\frac{p}{p-q-1}} .
$$

Notice that the first ones blow-up type solutions are half-space solutions and the last one is a radial solutions with dead core being precisely $B_{\mathfrak{R}_{0}}\left(x_{0}\right)$.

In the next paragraph, we shall be devoted to establish a general Liouville-type theorem for any entire solution to (1.2).

A Liouville-type Theorem Liouville-type results have represented an important chapter in the modern mathematical history mainly due to their quantitative and classificatory character. Such results have presented several implications in Analysis, PDEs, Geometry and free boundary problems. In effect, the knowledge of the asymptotic behaviour/global profile for certain entire solutions at infinity is a decisive information in many quantitative researches (cf. [19] for an interesting work on this direction).

In this part, we are concerned in proving a Liouville-type result for global dead-core solutions. In other words, a global weak solution must grow faster than $\max \{|x|, \sqrt[\theta]{|t|}\}^{\frac{p}{p-q-1}}$ as $\max \{|x|, \sqrt[\theta]{|t|}\} \rightarrow \infty$, unless it is identically zero. The next Theorem is based on [31, Theorem 8], thus we will include some details for completeness.

Theorem 5.4. Let $u$ be an entire weak solution to:

$$
\Delta_{p} u(x, t)-\frac{\partial u}{\partial t}(x, t)=\lambda_{0}(x, t) \cdot u_{+}^{q}(x, t)
$$

with $u(0,0)=0$ and $\lambda_{0}$ as before. If $u(x, t)=\mathrm{o}\left(\max \{|x|, \sqrt[\theta]{|t|}\}^{\frac{p}{p-q-1}}\right)$ as $\max \{|x|, \sqrt[\theta]{|t|}\} \rightarrow \infty$, then $u \equiv 0$.

Proof. For each positive number $r \gg 1$, let us define the auxiliary scaled function $u_{r}: Q_{1} \rightarrow \mathbb{R}_{+}$given by

$$
u_{r}(x, t):=\frac{u\left(r x, r^{\theta} t\right)}{r^{\frac{p}{p-q-1}}} .
$$

Thus, it is easy to check that

$$
\Delta_{p} u_{r}-\frac{\partial u_{r}}{\partial t}=\lambda_{0}\left(r x, r^{\theta} t\right)\left(u_{r}\right)_{+}^{q} \quad \text { in } \quad Q_{1}
$$

in the weak sense and $u_{r}(0,0)=0$. Now, we affirm that

$$
\left\|u_{r}\right\|_{L^{\infty}\left(Q_{1}\right)}=\mathrm{o}(1) \quad \text { as } \quad r \rightarrow \infty .
$$

In effect, for each $r \in \mathbb{R}_{+}$, let $\left(x_{r}, t_{r}\right) \in \mathbb{R}^{N} \times \mathbb{R}$ be such that $u_{r}$ achieves its maximum, i.e.,

$$
u_{r}\left(x_{r}, t_{r}\right)=\sup _{Q_{1}} u_{r}(x, t) \text {. }
$$

Now, we must analyse two possibilities: 
(1) If $\lim _{r \rightarrow \infty} \max \left\{\left|r x_{r}\right|, \sqrt[\theta]{\left|r^{\theta} t_{r}\right|}\right\}=\infty$, we obtain by using the assumption

$$
\begin{aligned}
u_{r}\left(x_{r}, t_{r}\right) & =\frac{u\left(r x_{r}, r^{\theta} t_{r}\right)}{\max \left\{\left|r x_{r}\right|, \sqrt[\theta]{\left|r^{\theta} t_{r}\right|}\right\}^{\frac{p}{p-q-1}}} \max \left\{\left|x_{r}\right|, \sqrt[\theta]{\left|t_{r}\right|}\right\}^{\frac{p}{p-q-1}} \\
& \leq C(N, p, q) . o(1) \rightarrow 0 \text { as } \quad r \rightarrow \infty .
\end{aligned}
$$

(2) On the other hand, if $\lim _{r \rightarrow \infty} \max \left\{\left|r x_{r}\right|, \sqrt[\theta]{\left|r^{\theta} t_{r}\right|}\right\}<\infty$ we derive the same conclusion as before for $u_{r}\left(x_{r}, t_{r}\right)$, since $u$ is a continuous function (cf. [18], [19], and [34] for regularity of in-homogeneous degenerate evolution equations).

Therefore, by applying Theorem 1.2 we obtain

$$
\begin{aligned}
u_{r}(x, t) & \leq \mathrm{o}(1) \cdot \mathfrak{C}\left(N, p, q, \lambda_{0}\right)\left(|x|+|t| \frac{1}{\theta}\right)^{\frac{p}{p-q-1}} \\
& \leq \mathrm{o}(1) \cdot \mathfrak{C}\left(N, p, q, \lambda_{0}\right) \max \{|x|, \sqrt[\theta]{|t|}\}^{\frac{p}{p-q-1}} \quad \text { in } \quad Q_{\frac{1}{2}} \quad \text { for } \quad r \gg 1,
\end{aligned}
$$

where we have used the equivalence of norms in $\mathbb{R}^{N+1}$. Now, suppose reach a contradiction that there exists a $\left(x_{0}, t_{0}\right) \in\left(\mathbb{R}^{N} \times \mathbb{R}_{+}\right) \backslash\{(0,0)\}$ such that $u\left(x_{0}, t_{0}\right)>0$. Observe that (5.2) means that given $\xi>0$ there exists an $r_{0}=r_{0}(\xi, p, q)>0$ such that

$$
\sup _{Q_{\frac{1}{2}} \max \{|x|, \sqrt[\theta]{|t|}\}^{\frac{p}{p-q-1}}} \leq \xi
$$

provided $r>r_{0}$. For such a $\xi$ fixed, we now estimate, for $r \gg 2 \max \left\{\left|x_{0}\right|, \sqrt[\theta]{\left|t_{0}\right|}, r_{0}\right\}$ :

$$
\frac{u\left(x_{0}, t_{0}\right)}{\max \left\{\left|x_{0}\right|, \sqrt[\theta]{\left|t_{0}\right|}\right\}^{\frac{p}{p-q-1}}} \leq \sup _{Q_{\frac{r}{2}}} \frac{u(x, t)}{\max \{|x|, \sqrt[\theta]{|t|}\}^{\frac{p}{p-q-1}}} \leq \sup _{Q_{\frac{1}{2}} \max \{|x|, \sqrt[\theta]{|t|}\}^{\frac{p}{p-q-1}}} \leq \xi .
$$

The proof finishes by selecting $0<\xi<\frac{u\left(x_{0}, t_{0}\right)}{\max \left\{\left|x_{0}\right|, \sqrt[\theta]{\left|t_{0}\right|}\right\}^{\frac{p}{p-q-1}}}$, which is possible due to our hypothesis. Such a contradiction completes the proof of Theorem.

Remark 5.5. Consider:

for $i=1, \cdots, N$, where

$$
u(x, t)=\left\{\begin{array}{l}
{\left[\lambda_{0}(1-q)(-t)\right]_{+}^{\frac{1}{1-q}}} \\
C_{p, q} \cdot\left(x_{i}\right)_{ \pm}^{\frac{p}{p-1-q}} \\
C_{p, q} \cdot\left(\left|x-x_{0}\right|-\Re_{0}\right)_{+}^{\frac{p}{p-1-q}}
\end{array}\right.
$$

$$
C_{p, q}:=\left(\lambda_{0} \cdot \frac{(p-q-1)^{p}}{p^{p-1}(p q+N(p-1-q))}\right)^{\frac{1}{p-q-1}}
$$

Then, $u$ is a weak solutions to

$$
\Delta_{p} u(x, t)-\frac{\partial u}{\partial t}(x, t)=\lambda_{0} \cdot u_{+}^{q}(x, t) .
$$

Then, the assumption of Theorem 5.4 is sharp in the sense that there are half-space/ radial solutions such that

$$
\frac{u(x, t)}{\left(\max \{|x|, \sqrt[\theta]{|t|}\}^{\frac{p}{p-q-1}}\right)}>0 \quad \text { as } \quad \max \{|x|, \sqrt[\theta]{|t|}\} \rightarrow \infty .
$$

For an analysis about radial solutions of fully non-linear elliptic and quasi-linear dead-core problems we recommend [11, Section 6] and [13, Section 5.2]. 


\section{ACKNOWLEDGEMENTS}

This work was partially supported by ANPCyT under grant PICT 2012 - 0153, by Consejo Nacional de Investigaciones Científicas y Técnicas (CONICET-Argentina PIP 5478/1438), by the CNPq (Brazilian Government Program Ciência sem Fronteiras) and by Universidad de Buenos Aires under grant UBACYT 20020100100400. The authors would like to thank Prof. Jesús Ildefonso Díaz for pointing out many valuable references. The authors would also like to thank Prof. Noemí Wolanski for several insightful comments and discussions. The authors are also grateful to the anonymous referee(s) for pointing out a number of improvements that benefited a lot the final outcome of the article. JV da Silva thanks IMASL (CONICET) from Universidad Nacional de San Luis for its warm hospitality and for fostering a pleasant scientific atmosphere during his visit where part of this paper was written. JV da Silva also thanks (CONICET - Postdoctoral Fellowship) and Research Group of PDEs/Math. Dept./FCEyN from Universidad de Buenos Aires by the excellent working environment during his Postdoctoral program. P. Ochoa and A. Silva were partially supported by CONICET-Argentina.

\section{REFERENCES}

[1] ÁLVAREZ, L. and DÍAZ, J. I. "On the initial growth of interfaces in reaction-diffusion equations with strong absorption". Proceedings of the Royal Society of Edinburgh 123 A, 803-817, 1993 .

[2] ANDERSON, J., LINDGREN, E. and SHAHGHOLIAN, H. "Optimal regularity for the obstacle problem for the $p$-Laplacian”. J. Differential Equations, 259 (2015), no. 6, 2167-2179.

[3] ANTONTSEV, S. N.; DÍAZ, J. I. and SHMAREV, S. "The support shrinking properties for solutions of quasilinear parabolic equations with strong absorption terms". Annales de la Faculté des Sciences de Toulouse IV no. 1, 5-30, 1995.

[4] ANTONTSEV, S. N. DÍAZ, J. I. and SHMAREV, S. "Energy methods for free boundary problems. Applications to nonlinear PDEs and Fluid Mechanics". Series Progress in Nonlinear Differential Equations and Their Applications, N. 48, Birkäuser, Boston, 2002.

[5] ARAÚJO, D. J., TEIXEIRA, E. V. and URBANO, J. M. A proof of the $C^{p^{\prime}}$ regularity conjecture in the plane. Adv. Math. 316 (2017), $541-553$.

[6] ARAÚJO, D. J., TEIXEIRA, E. V. and URBANO, J. M. Towards the $C^{p^{\prime}}$ regularity conjecture. To appear in Int. Math. Res. Not. https: //doi . org/10 . 1093/imrn/rnx068.

[7] BANDLE, C. and STAKGOLD, I. "The formation of the dead core in parabolic reaction-diffusion problems". Transactions of the American Mathematical Society 286 no. 1, 275-293, 1984

[8] CAFFARELLI, L.A. and SALSA, S. "A Geometric Approach to Free Boundary Problems". Grad. Stud. in Math. 68, Amer. Math. Soc., Providence, RI, 2005.

[9] CHOE, H.J. and WEISS, G. S. "A semilinear parabolic equation with free boundary". Indiana Univ. Math. J., 52 no. 1, 19-50, 2003.

[10] DA SILVA, J. V. and DOS PRAZERES, D. S. "Schauder Type Estimates for "Flat" Viscosity Solutions to Non-convex Fully Nonlinear Parabolic Equations and Applications". To appear in Potential Anal (2017). https://doi.org/10.1007/s11118-017-9677-z.

[11] DA SILVA, J. V.; LEITÃO, R. A. and RICARTE, G. C. "Fully nonlinear elliptic equations of degenerate/singular type with free boundaries". Submitted.

[12] DA SILVA, J. V. and OCHOA, P. "Fully nonlinear parabolic dead core problems". Submitted.

[13] DA SILVA, J. V. and SALORT, A.. "Regularity estimates for quasi-linear elliptic equations with free boundaries". Submitted.

[14] DA SILVA, J. V. and TEIXEIRA, E.V. "Sharp regularity estimates for second order fully nonlinear parabolic equations". Math. Ann. 369 (2017), no. 3-4, 1623-1648, https://doi.org/10.1007/s00208-016-1506-y.

[15] DÍAZ, J. I. "Qualitative Study of Nonlinear Parabolic Equations: an Introduction”. Extracta Mathematicae 16 no. 2, 303-341, 2001.

[16] DÍAZ, J.I. and HERRERO, M. "Propriétés de support compact pour certaines équations elliptiques et paraboliques non linéaires". C.R. Acad. Sc. Paris 286 Série I, 815-817, 1978.

[17] DÍAZ, J.I. and MINGAZZINI, T. "Free boundaries touching the boundary of the domain for some reaction-diffusion problems". Nonlinear Analysis Series A: Theory, Mehods and Applications 119, 275-294, 2015.

[18] DIBENEDETTO, E. "Degenerate parabolic equations". Springer-Verlag, New York, 1993.

[19] DIBENEDETTO, E.; GIANAZZA, U. and VESPRI, V. "Liouville-type theorems for certain degenerate and singular parabolic equations". C. R. Math. Acad. Sci. Paris 348, 873-877, 2010.

[20] DIBENEDETTO, E.; URBANO, J. M. and VESPRI, V. "Current issues on singular and degenerate evolution equation". Handbook of Differential Equations $1,169-286,2002$

[21] GUO, J-S. "On the dead-core rates for a parabolic equation with strong absorption". Bulletin of the Institute of Mathematics Academia Sinica (New Series) 8 no. 4, 431-444, 2013

[22] KARP, L., KILPELÄINEN, T. PETROSYAN, A. and SHAHGHOLIAN, H. "On the porosity of free boundaries in degenerate variational inequalities". J. Differential Equations 164 (2000), no. 1, 110-117.

[23] PETROSYAN, A. and SHAHGHOLIAN, H. "Parabolic obstacle problems applied to finance: free-boundary-regularity approach". Editor D. Danielle, Recent Developments in Nonlinear Partial Differential Equations, Contemporary Matematics 439, 2007, 117-133 (Appendix by T. Arnarson).

[24] PRAZERES, D. and TEIXEIRA, E.V. "Asymptotics and regularity of flat solutions to fully nonlinear elliptic problems". Ann. Sc. Norm. Super. Pisa Cl. Sci. Vol. XV (2016), 485-500

[25] SHAHGHOLIAN, H. "Analysis of the Free Boundary for the $p$-parabolic variational problem $p \geq 2$ ". Rev. Mat. Iberoamericana, 19 no. 3, 797-812, 2003.

[26] SHAHGHOLIAN, H. "Free boundary regularity close to initial state for parabolic obstacle problem". Trans. Amer. Math. Soc. 360, 2077-2087, 2007.

[27] SPERB, R. "Some complementary estimates in the dead core problem". SIAM J. of Math. Analysis. Special Issue in Honor of I. Stalegold. 1996

[28] STAKGOLD, I. "Reaction-diffusion problems in chemical engineering". Nonlinear diffusion problems (Montecatini Terme, 1985), Lecture Notes in Math. 1224, Springer, Berlin, 1986, pp. 119-152.

[29] TEIXEIRA, E. V. Regularity for quasilinear equations on degenerate singular set. Math. Ann. 358 (2014), 241-256.

[30] TEIXEIRA, E. V. "Geometric regularity estimates for elliptic equations". Proceedings of the MCA 2013 Contemp. Math. 656, 185-204, 2016.

[31] TEIXEIRA, E.V. "Regularity for the fully nonlinear dead-core problem". Math. Ann. 364, no. 3-4, 1121-1134, 2016.

[32] TEIXEIRA, E.V. "Nonlinear elliptic equations with high order singularities". To appear in Potential Anal (2017). https://doi.org/10.1007/ S1118-017-9637-7.

[33] TEIXEIRA, E. V. and URBANO, J. M. "A geometric tangential approach to sharp regularity for degenerate evolution equations". Anal. PDE. 7 no. 3, $733-744$, 2014. 
[34] URBANO, J. M. "The Method of Intrinsic Scaling - A Systematic Approach to Regularity for Degenerate and Singular PDEs". Lectures Notes in Mathematics 1930, Springer-Verlag, Berlin, 2008

[35] VÁZQUEZ, J. L. "A strong maximum principle for some quasilinear elliptic equations". Appl. Math. Optim. 12, 191-202, 1984

[36] ZAJICEK, L. "Porosity and $\sigma$-porosity". Real Anal. Exchange 13, 314-350, 1987.

(João Vitor da Silva) Universidad de Buenos Aires. Departamento de Matemática - FCEyN - CiUdad Universitaria, Pabellón I (1428). Buenos Aires, Argentina.

(Pablo Ochoa) Universidad Nacional de Cuyo. Universidad Nacional de SAn Luis and CONICET, Mendoza 5500, ARGENTINA

(Analia Silva) Instituto de Matemática Aplicada SAn Luis-IMASL. Universidad Nacional DE SAN LUIS ANd CONICET. EJERCito de los Andes 950. D5700HHW SAn Luis. Argentina.

E-mail address, J.V. da Silva: jdasilva@dm. uba. ar

E-mail address, P. Ochoa: ochopablo@gmail.com

E-mail address, A. Silva: asilva@dm. uba . ar 